\title{
Additive influences of maternal and paternal body mass index on weight status trajectories from childhood to mid- adulthood in the 1970 British Cohort Study
}

\author{
Silvia Costa Institute of Child Health, University College London, UK \\ silvia.costa@ucl.ac.uk \\ William Johnson MRC Human Nutrition Research Unit, Cambridge, UK \\ Russell M Viner Institute of Child Health, University College London, UK
}

(Received July 2014 Revised February 2015)

http://dx.doi.org/10.14301/Ilcs.v6i2.301

\section{Abstract}

This study aimed to (i) describe the weight status trajectories from childhood to mid-adulthood and (ii) investigate the influence of maternal and paternal body mass index (BMI) on offspring's trajectories in a nationally representative study in Great Britain. The sample comprised 4,174 (43\% male) participants from the 1970 British Cohort Study with complete BMI data at ages 10, $26,30,34$, and 42 years. Individuals' weight status was categorised as overweight/obese or non-overweight/obese at each age, and trajectories of weight status from 10 to 42 years of age were assessed. Sex-stratified multinomial logistic regression models were used to assess associations of maternal and paternal BMI with trajectory group membership, adjusting for potential confounders (e.g. socioeconomic position and puberty). Thirty per cent of individuals were never overweight/obese (reference trajectory), 6\%, 44\% and $8 \%$ had childhood, early-and mid-adulthood onset of overweight/obesity (respectively), and 12\% other trajectories. In fully adjusted models, higher maternal and paternal BMI significantly increased the risk of childhood (relative risk ratio: 1.2-1.3) and early adulthood onset (1.2) of overweight/obesity in both sexes. Relative risk ratios were generally higher for maternal than paternal BMI in females but similar in males. Early puberty also increased the risk of childhood (1.8-9.2) and early-adulthood onset (3.7-4.7) of overweight/obesity. Results highlight the importance of primary prevention, as most individuals remained overweight/obese after onset. Maternal and paternal BMI had additive effects on offspring weight status trajectories across 32 years of the life course, suggesting that prevention/intervention programmes should focus on the whole family.

Keywords: Obesity; trajectories; tracking; life course; parental BMI; socioeconomic factors

\section{Introduction}

The obesity epidemic is one of the greatest public health concerns worldwide (World Health Organization, 2011; Health and Social Care Information Centre, 2014a). The increase in the prevalence of obesity has been seen in both adults and children (World Health Organization, 2011). In England the prevalence of overweight and obesity in adults has increased from $58 \%$ and $49 \%$ in 1993 to $67 \%$ and $57 \%$ in men and women respectively in
2012 (Health and Social Care information Centre, 2014a). Overweight and obesity in children has also increased, with peaking rates of $18 \%$ and $19 \%$ of 2 15 year-old boys and girls (respectively) in the 2004 Health Survey for England (Health and Social Care Information Centre, 2014a). Although rates in the Health Survey for England seem to be stabilising in recent years (Health and Social Care Information Centre, 2014a), other data sources show different rates and trends of childhood overweight and 
obesity. For example, the latest sweep of the National Childhood Measurement Programme has reported that over a fifth of children in reception year (5-6 years) and about a third of those in year six (10-11 years) were overweight or obese, and that these rates were higher than those reported for the previous school year (Health and Social Care Information Centre, 2014b). This is worrying since obesity has been shown to track from childhood through to adulthood (Reilly et al., 2003; Venn et al., 2007; Brisbois, Farmer, \& McCargar, 2012), and both childhood and adulthood obesity are associated with adverse health outcomes (Park, Falconer, Viner, \& Kinra, 2012; Park, Sovio, Viner, Hardy, \& Kinra, 2013; Prospective Studies Collaboration, 2009; Reilly et al, 2003).

Parental body mass index (BMI) is one of the most consistent factors associated with offspring obesity (Monasta et al., 2010; Parsons, Power, Logan, \& Summerbell, 1999). For example, using data from the 1958 British birth cohort, Power, Manor and Matthews (2003) found a significant association of higher maternal and paternal BMI with obesity at 33 years, even when controlling for adulthood (in males) and childhood socio-economic position (SEP), and participants' education at 33 years. However, most research to date has only considered obesity at one time-point in adulthood (Parsons et al., 1999), and the influence of parental BMI on different trajectories of weight status (e.g. persistent overweight/obesity from childhood to adulthood) remains largely unknown. This consistent association of higher parental BMI with offspring overweight/obesity has been hypothesised to operate through a variety of different genetic (e.g. heritance of "obesity genes") (Maes, Neale, \& Eaves, 1997), biological (e.g. restricted or excessive intrauterine growth), and shared environmental factors (e.g. inadequate diet and sedentary lifestyles) (Monasta et al., 2010; Parsons, et al., 1999). Few studies have investigated the association of maternal and paternal BMI with offspring overweight and obesity later than earlyadulthood (Parsons, et al., 1999), and much of the existing literature suffers from potential confounding effects that were not accounted for, such as SEP and early maturation (Monasta et al., 2010).

Identifying the exact mechanisms through which parental obesity leads to later obesity is difficult, because of the many possible mediators and confounders involved in this relationship (Monasta et al., 2010; Parsons et al., 1999). It is important to clarify whether parental BMI has a significant influence in their offspring's lifelong weight status trajectories, given the high prevalence of adult overweight/obesity (World Health Organization, 2011; Health and Social Care Information Centre, 2014a) and that it is potentially easier to directly intervene in parents than in their offspring (e.g. through change in diet and physical activity) (Golan, 2006; Monasta et al, 2010). Studying large samples where basic measures of the risk factors have been collected has been suggested as a potential approach to deal with the inherent difficulty in measuring the risk factors associated with overweight/obesity (Parsons et al., 1999). The 1970 British Cohort Study (BCS70) has recently released the data for the 42-year follow-up; this presents a remarkable opportunity to investigate the influence of parental BMI on offspring's weight status trajectories from childhood to mid-adulthood, addressing some of the main limitations of previous studies - long-term follow-up (birth to 42 years) and data available for several confounders (e.g. SEP, breast-feeding, and maturation).

The aims of this study were to (i) describe weight status trajectories from childhood to mid-adulthood in the BCS70 and (ii) investigate the separate influences of maternal and paternal BMI on offspring's weight status trajectories.

\section{Methods}

\section{Sample}

The BCS70 has followed just under 17,200 people born in Great Britain (England, Scotland, and Wales) in one week in April 1970, from birth onwards (Centre for Longitudinal Studies, 2014). Nine main data collections have taken place: at birth (1970), 5 (1975), 10 (1980), 16 (1986), 26 (1996), 30 (2000), 34 (2004), 38 (2008), and 42 (2012) years of age. At the most recent sweep $57 \%(N=9,842)$ of the participants were still in the study and they remained broadly representative of the national population of men and women of the same age. Participants were eligible for this study if they provided valid BMI data at age 42 years $(n=8,799)$, and were included if they also had valid BMI data at 10,26 or 30,34 , and 42 years of age. The final 
sample for the present study comprised 4,174 (1,782 male; 2,392 female) participants, representing $47 \%$ of the 8,799 eligible participants. This sample was not noticeably different from the remaining 4,625 (53\%) eligible participants who did not meet the inclusion criteria for the present study. There were no significant differences in parental $\mathrm{BMI}$, and only small differences between included and excluded participants in some of the other variables: breastfeeding $\geq 3$ months ( $87 \%$ of included versus $90 \%$ of excluded); early puberty ( $17 \%$ of included versus $15 \%$ of excluded); high SEP (34\% of included versus $30 \%$ of excluded) (all $p<0.001)$.

\section{Participants' body mass index}

Weight and height were measured by community medical officers, health visitors or school nurses at 10 and 16 years of age according to standard protocols, and self-reported in questionnaires at 26 years and face-to-face interviews at 30,34, and 42 years of age. Due to the low response rate, the amount of data collected at age 16 was considerably less than at all other ages (e.g. valid $\mathrm{BMI}: 5,723$ versus $\geq 7,303$ participants). This was due to prolonged industrial action by teachers in 1986, who were responsible for the educational tests; the delayed survey start meant that many children had left school before assessments could be done and information for many of them could not be obtained. Therefore, data from the 16-year follow-up was excluded from this study. Body mass index (weight $(\mathrm{kg}) /$ height $\left.(m)^{2}\right)$ was computed at ages $10,26,30,34$, and 42 years. Due to the small number of obese participants at each age, weight status was categorised as either " 1 = overweight/obese" or " 0 = non-overweight/obese". In adulthood, participants' BMI was classified as overweight/obese when $\geq 25 \mathrm{~kg} / \mathrm{m}^{2}$, and nonoverweight/obese if $<25 \mathrm{~kg} / \mathrm{m}^{2}$. At 10 years of age, the International Obesity Task Force BMI cut-off points for overweight (Cole, Bellizzi, Flegal, \& Dietz, 2000) were used to classify cohort members as overweight/obese or non-overweight/obese. To maximise sample size, the 26 and 30 year surveys were collapsed into one time-point, as the 26 year survey had substantially less valid BMI data and these two time points were closely spaced. Participants who were overweight/ obese at either age or both ages were coded as overweight/obese, and participants who were normal weight at both ages were coded as normal weight.

\section{Parental body mass index and confounding variables}

Maternal and paternal weight ( $\mathrm{kg}$ or stones and pounds) and height (meters or feet and inches) were reported by the mother when cohort member was 10 years of age. Data in stones/pounds and feet/inches were transformed into $\mathrm{kg}$ and meters respectively, and parental BMI was subsequently calculated and used as a continuous variable in all analyses. Birth weight (in grams) and gestational age at birth (in completed weeks) was recorded by the midwife who had undertaken the delivery or the senior midwife. As there is good evidence of a positive relation between high birth weight and higher fatness levels (Parsons et al., 1999), birth weight was classified as "high birth weight" if $>4 \mathrm{~kg}$, or "medium-low birth weight" if $\leq 4 \mathrm{~kg}$. Duration of breast-feeding was reported by the mother as "never breastfed", "<1 month", " $\geq 1$ month but $<3$ months", and " $\geq 3$ months", when participants were aged 5 years. A binary breast-feeding variable with the responses " $<3$ months" or " $\geq 3$ months" was computed. Evidence of puberty at age 10 years was assessed and recorded during the medical examination by a community medical officer, a health visitor or the school nurse, by answering "yes"/"no" to the question "Did your examination reveal (...) Any evidence of puberty?". Maternal and paternal occupation was reported by the mother at the 10 year follow-up, and classified according to the 1980 national Classification of Occupations (Office of Population Censuses and Surveys, 1980). Because higher parental SEP and education have shown some evidence of being protective of offspring obesity (Monasta et al., 2010), SEP and maternal education variables were dichotomised as follows. A binary SEP variable with the responses "high SEP" (if the mother, father, or both belonged to a professional or managerial social class) or "medium-low SEP" (if both parents belonged to skilled, partly-skilled or unskilled social classes) was derived. Maternal education was also reported by the mother at the 10 year follow-up, and a binary variable comprising "A-levels or above" (A-levels are the requirement for university access) and "below A-levels" was computed. 


\section{Weight status trajectories}

Weight status trajectories between 10 and 42 years of age were defined manually for males and females separately, using a three-step procedure. We first tabulated all possible combinations of the binary weight status variable across the four ages to produce 16 trajectories (i.e. $2^{4}$ ) and examined the frequency of participants belonging to each trajectory (see appendix 1). Second, all trajectories comprising $<5 \%$ of the sample were collapsed into one group named "other trajectories", and those comprising $\geq 5 \%$ of the sample were retained as individual trajectories, namely: "never overweight/obese", comprising individuals who were never classified as overweight/obese between 10 and 42 years; "mid-adulthood onset of overweight/obesity", comprising individuals who were overweight/obese only at age 42 years; "overweight/obesity onset at age 34 years", comprising individuals who were overweight/obese from 34 years onwards; "overweight/obesity onset at ages 26 or 30 years", comprising individuals who were overweight/obese from 26-30 years onwards; and "childhood onset of overweight/obesity", comprising individuals who were always overweight/obese between 10 and 42 years of age. Third, to improve power and because both trajectories represented two timings of early adulthood, the trajectories "overweight/obesity onset at age 34 years" and "overweight/obesity onset at ages 26 or 30 years" were collapsed into a single trajectory re-labelled as "early-adulthood onset of overweight/obesity". The final outcome variable consisted of five trajectories: 1) "never overweight/obese", 2) "mid-adulthood onset of overweight/obesity", 3) "early-adulthood onset of overweight/obesity", 4) "childhood onset of overweight/obesity", and 5) "other trajectories".

\section{Statistical Analyses}

Descriptive statistics were used to describe the sample and the trajectories of weight status. All continuous variables were tested for normality of distribution with the Shapiro-Francia test and found to be non-normally distributed. Mann-Whitney $U$ tests were used to assess differences in continuous and ordinal variables between sexes, and between those included and excluded from analyses. Chisquare tests were used to assess differences in categorical variables between sexes, and between those included and excluded from analyses.

Analyses were conducted separately for males and females. This was to test whether parental BMI affects weight status trajectories differently in males and females, and because of the sex inequality in the consequences of overweight /obesity, the potential differences in the association of early life factors (such as SEP) with adulthood overweight/obesity (Heraclides, Witte, \& Brunner, 2008; Khlat, Jusot \& Ville, 2009; Pudrovska, Logan \& Richman, 2014), and the significant differences in the prevalence of weight status trajectories. Univariable multinomial logistic regression was used to assess the associations of maternal and paternal BMI (separately) with weight status trajectory membership. Multivariable multinomial logistic regression was then used to test how these associations change after adjusting for the confounding effect of birth factors (step 1) and puberty (step 2), maternal education and SEP (step 3). This was firstly done for maternal and paternal BMI separately, and subsequently with maternal and paternal $\mathrm{BMI}$ in the same model. Interactions between maternal and paternal BMI were also tested. To assess whether the results on the associations of parental BMI with offspring's weight status trajectories may be influenced by the different distributions of maternal and paternal BMI values, post-hoc sensitivity analyses were undertaken using parental BMI z-score (zBMI) instead of absolute BMI. Alpha-value was set at 0.05, with Bonferroni correction applied to multiple comparisons. All analyses were conducted in Stata (v.12) statistical software package.

\section{Results}

Details of the 4,174 participants included for analyses can be seen below in Table 1 . More females $(57.3 \%)$ than males were included for analyses. Significantly more females showed evidence of puberty at 10 years than males $(p<0.001)$, whereas males had significantly higher birth weight and BMI at 42 years of age than females $(p<0.001)$. All other variables were broadly similar for both sexes. 


\section{Table 1. Description of study sample}

\begin{tabular}{|c|c|c|c|c|c|c|}
\hline & $\begin{array}{c}\text { Missing data } \\
{\left[\mathrm{N}\left(\%^{1}\right)\right]}\end{array}$ & & $\begin{array}{c}\text { Total } \\
(\mathrm{N}=\mathbf{4 , 1 7 4})\end{array}$ & $\begin{array}{c}\text { Females } \\
(N=2,392)\end{array}$ & $\begin{array}{c}\text { Males } \\
(\mathrm{N}=1,782)\end{array}$ & $\begin{array}{c}p \\
\text { (sex difference) }\end{array}$ \\
\hline $\mathrm{BMI}$ at 10 years $\left(\mathrm{kg} / \mathrm{m}^{2}\right)$ & $0(0.0)$ & Median (IQR) & $16.7(15.6-18.1)$ & $16.7(15.5-18.3)$ & $16.7(15.7-18.1)$ & 0.605 \\
\hline $\mathrm{BMI}$ at 42 years $\left(\mathrm{kg} / \mathrm{m}^{2}\right)$ & $0(0.0)$ & Median (IQR) & $26.6(23.6-30.1)$ & $25.2(22.6-29.4)$ & $27.9(25.5-30.7)$ & $<0.001$ \\
\hline Gestational age (weeks) & $864(20.7)$ & Median (IQR) & $40(39-41)$ & $40(39-41)$ & $40(39-41)$ & 0.016 \\
\hline Birth weight $(\mathrm{kg})$ & $241(5.8)$ & Median (IQR) & $3.3(3.0-3.6)$ & $3.3(3.0-3.6)$ & $3.4(3.1-3.7)$ & $<0.001$ \\
\hline Medium-low birth weight & & $\mathrm{N}\left(\right.$ valid $\left.\%{ }^{2}\right)$ & $3617(92.0)$ & 134 (5.9) & $182(10.9)$ & \\
\hline High birth weight & & $\mathrm{N}\left(\right.$ valid $\left.\%{ }^{2}\right)$ & $316(8.0)$ & $2130(94.1)$ & $1487(89.1)$ & \\
\hline Breast-feeding duration & $569(13.6)$ & & & & & 0.466 \\
\hline$<3$ months & & $\mathrm{N}\left(\right.$ valid $\left.\%^{2}\right)$ & $3,127(86.7)$ & $1,796(86.4)$ & $1,331(87.2)$ & \\
\hline$\geq 3$ months & & $\mathrm{N}\left(\right.$ valid $\left.\%^{2}\right)$ & $478(13.3)$ & $283(13.6)$ & $195(12.8)$ & \\
\hline Evidence of puberty at 10 years & $18(0.4)$ & & & & & $<0.001$ \\
\hline No & & $\mathrm{N}\left(\right.$ valid $\%{ }^{2}$ ) & $3,432(82.6)$ & $1,735(72.8)$ & $1,697(95.7)$ & \\
\hline Yes & & $\mathrm{N}\left(\right.$ valid $\left.\%^{2}\right)$ & $724(17.4)$ & $647(27.2)$ & $77(4.3)$ & \\
\hline Maternal BMI $\left(\mathrm{kg} / \mathrm{m}^{2}\right)$ & $116(2.8)$ & Median (IQR) & $22.6(21.0-25.0)$ & $22.5(21.0-24.9)$ & $22.7(21.1-25.0)$ & 0.352 \\
\hline Paternal BMI $\left(\mathrm{kg} / \mathrm{m}^{2}\right)$ & $274(6.6)$ & Median (IQR) & $24.1(22.5-26.0)$ & $24.1(22.5-26.0)$ & $24.1(22.5-26.3)$ & 0.437 \\
\hline Maternal education at 10 years & $251(6.0)$ & & & & & 0.271 \\
\hline Less than A-level & & $\mathrm{N}\left(\right.$ valid $\left.\%^{2}\right)$ & $3,184(81.2)$ & $1,825(80.6)$ & $1,359(82.0)$ & \\
\hline A-level or above & & $\mathrm{N}\left(\right.$ valid $\left.\%{ }^{2}\right)$ & $739(18.8)$ & $440(19.4)$ & $299(18.0)$ & \\
\hline Parental SEP at 10 years & $114(2.7)$ & & & & & 0.453 \\
\hline Medium-low & & $\mathrm{N}\left(\right.$ valid $\%{ }^{2}$ ) & $2,711(66.8)$ & $1,542(66.3)$ & $1,169(67.4)$ & \\
\hline High & & $\mathrm{N}\left(\right.$ valid $\left.\%^{2}\right)$ & $1,349(33.2)$ & $784(33.7)$ & $565(32.6)$ & \\
\hline
\end{tabular}

Legend: BMI - Body mass index; IQR - Inter-quartile range; SEP - Socio-economic position.

${ }^{1}$ percent of total $(4,174)$ participants with trajectories of weight status.

2 percent of total $(4,174)$ participants with valid data for each variable. 


\section{Trajectories of weight status}

Significantly more females than males were never overweight/obese and had mid-adulthood onset of overweight/obesity $(p<0.001)$, whereas the prevalence of males having early-adulthood onset of overweight/obesity was roughly double the prevalence of females $(62.7 \%$ versus $30.6 \%$; $p<0.001)$. Prevalence of childhood onset of overweight/obesity was the same in females and males (see table 2). Importantly, of those overweight/obese in childhood (229 females; 129 males), the wide majority remained overweight/ obese in at least two time points during adulthood - $85 \%$ of females and $92 \%$ of males (see table in appendix 1).

Table 2. Description of trajectories of weight status included for regression analyses [n (\%)].

\begin{tabular}{cccc}
\hline & $\begin{array}{c}\text { Total } \\
(\mathrm{n}=4,174)\end{array}$ & $\begin{array}{c}\text { Females } \\
(\mathrm{n}=2,392)\end{array}$ & $\begin{array}{c}\text { Males } \\
(\mathrm{n}=1,782)\end{array}$ \\
\hline Never OW/OB & $1,230(29.5 \%)$ & $962(40.2 \%)$ & $268(15.0 \%)^{*}$ \\
$\begin{array}{c}\text { Mid-adulthood } \\
\text { OW/OB onset }\end{array}$ & $334(8.0 \%)$ & $231(9.6 \%)$ & $103(5.8 \%)^{*}$ \\
$\begin{array}{c}\text { Early adulthood } \\
\text { OW/OB onset }\end{array}$ & $1,850(44.3 \%)$ & $733(30.6 \%)$ & $1117(62.7 \%)^{*}$ \\
$\begin{array}{c}\text { Childhood OW/OB } \\
\text { onset }\end{array}$ & $258(6.2 \%)$ & $148(6.2 \%)$ & $110(6.2 \%)$ \\
$\begin{array}{c}\text { Other trajectories } \\
\text { ow }\end{array}$ & $502(12.0 \%)$ & $318(13.3 \%)$ & $184(10.3 \%)$ \\
\hline
\end{tabular}

Legend: OW/OB - Overweight or obese; ${ }^{*} p<0.001$ for difference between sexes.

\section{The influence of parental BMI on trajectories of weight status}

In females, both maternal and paternal BMI were significantly and positively associated with increased risk of childhood, early- and midadulthood onset of overweight/obesity, and having "other trajectories" versus never being overweight/obese in unadjusted regression models (table 3). In males, higher maternal BMI was associated with increased risk of having childhood and early-adulthood onset of overweight/obesity, and of belonging to "other trajectories" versus never being overweight/obese. Higher paternal BMI was associated with increased risk of having childhood and early-adulthood onset of overweight/obesity versus never being overweight/ obese in unadjusted regression models (table 4). These associations remained significant for males and females even when considering both maternal and paternal BMI in the same model (unadjusted for other variables). Interaction between maternal and paternal BMI was not significant (results not shown) and, therefore, was not included in the subsequent multivariable regression models. Because results of the multivariable regression models were similar when analyses where conducted with maternal and paternal BMI separately and in the same model, only the results for the models including both maternal and paternal BMI are presented below (tables 3 and 4). Results for the multivariable regression models with maternal BMI only and paternal BMI separately can be seen in the tables presented in appendices 2-5.

\section{Results from fully adjusted models}

In females after adjustment for birth factors, puberty and SEP factors (table 3), higher maternal $\mathrm{BMI}$ remained significantly associated with higher risk of childhood, early- and mid-adulthood onset of overweight/obesity, and of belonging to "other 
trajectories" compared with never being overweight/obese (relative risk ratio (RRR): 1.081.27; all $p \leq 0.017)$. Higher paternal BMI significantly increased the risk of childhood and early-adulthood onset of overweight/obesity, and belonging to "other trajectories" compared with never being overweight/obese (RRR: 1.11-1.21; all $p<0.001$ ). High SEP was associated with lower risk of childhood and early-adulthood onset of overweight/obesity (RRR: 0.50 and 0.75 respectively; both $p<0.04)$, whereas early puberty increased the risk of childhood (RRR: 3.68; $95 \% \mathrm{Cl}$ : 2.29-5.92) and early-adulthood onset of overweight/obesity (RRR: 1.75; 95\% Cl: 1.31-2.34), and of belonging to "other trajectories" (RRR: 2.30; 95\% Cl: 1.62-3.28) compared to never being overweight/obese (all $p<0.001$ ). No other significant associations were found. 
Table 3. Associations between parental BMI and trajectories of weight status in females.

\begin{tabular}{|c|c|c|c|c|c|c|c|c|c|c|c|}
\hline \multirow[b]{2}{*}{ Risk Factor } & \multirow[b]{2}{*}{$\begin{array}{c}\text { Included } \\
\mathrm{n}(\%)\end{array}$} & \multirow[b]{2}{*}{$\begin{array}{c}\text { Missing } \\
\text { n (\%) }\end{array}$} & \multirow{2}{*}{$\begin{array}{c}\text { Never } \\
\text { OW/OB } \\
\text { reference }\end{array}$} & \multicolumn{2}{|c|}{$\begin{array}{l}\text { Mid-adulthood onset } \\
\text { of OW/OB }\end{array}$} & \multicolumn{2}{|c|}{$\begin{array}{l}\text { Early-adulthood } \\
\text { onset of OW/OB }\end{array}$} & \multicolumn{2}{|c|}{$\begin{array}{c}\text { Childhood onset of } \\
\text { OW/OB }\end{array}$} & \multicolumn{2}{|c|}{ Other trajectories } \\
\hline & & & & $\begin{array}{c}\text { RRR } \\
(95 \% \mathrm{Cl})\end{array}$ & $p$ & $\begin{array}{c}\text { RRR } \\
(95 \% \mathrm{Cl})\end{array}$ & $p$ & $\begin{array}{c}\text { RRR } \\
(95 \% \mathrm{Cl})\end{array}$ & $p$ & $\begin{array}{c}\text { RRR } \\
(95 \% \mathrm{Cl})\end{array}$ & $p$ \\
\hline \multicolumn{12}{|l|}{$\begin{array}{l}\text { Unadjusted individual } \\
\text { models }\end{array}$} \\
\hline Maternal BMI & $\begin{array}{l}2,339 \\
(97.8)\end{array}$ & $\begin{array}{c}53 \\
(2.2)\end{array}$ & & $\begin{array}{c}1.10 \\
(1.05-1.15)\end{array}$ & $<0.001$ & $\begin{array}{c}1.21 \\
(1.17-1.25)\end{array}$ & $<0.001$ & $\begin{array}{c}1.31 \\
(1.25-1.37)\end{array}$ & $<0.001$ & $\begin{array}{c}1.17 \\
(1.13-1.22)\end{array}$ & $<0.001$ \\
\hline Paternal BMI & $\begin{array}{l}2,250 \\
(94.1)\end{array}$ & $\begin{array}{c}142 \\
(5.9)\end{array}$ & & $\begin{array}{c}1.09 \\
(1.03-1.15)\end{array}$ & 0.002 & $\begin{array}{c}1.17 \\
(1.12-1.21)\end{array}$ & $<0.001$ & $\begin{array}{c}1.29 \\
(1.21-1.36)\end{array}$ & $<0.001$ & $\begin{array}{c}1.15 \\
(1.10-1.21)\end{array}$ & $<0.001$ \\
\hline Mutually adjusted & $\begin{array}{l}2,230 \\
(93.2)\end{array}$ & $\begin{array}{l}162 \\
(6.8)\end{array}$ & & & & & & & & & \\
\hline Maternal BMI & & & & $\begin{array}{c}1.10 \\
(10.4-1.15)\end{array}$ & $<0.001$ & $\begin{array}{c}1.20 \\
(1.16-1.24)\end{array}$ & $<0.001$ & $\begin{array}{c}1.29 \\
(1.23-1.35)\end{array}$ & $<0.001$ & $\begin{array}{c}1.16 \\
(1.12-1.21)\end{array}$ & $<0.001$ \\
\hline Paternal BMI & & & & $\begin{array}{c}1.08 \\
(1.02-1.14)\end{array}$ & 0.006 & $\begin{array}{c}1.14 \\
(1.10-1.18)\end{array}$ & $<0.001$ & $\begin{array}{c}1.23 \\
(1.16-1.31)\end{array}$ & $<0.001$ & $\begin{array}{c}1.13 \\
(1.07-1.18)\end{array}$ & $<0.001$ \\
\hline $\begin{array}{l}\text { Adjusted for birth } \\
\text { factors, puberty and } \\
\text { socioeconomic position }\end{array}$ & $\begin{array}{l}1,567 \\
(65.5)\end{array}$ & $\begin{array}{c}825 \\
(34.5)\end{array}$ & & & & & & & & & \\
\hline Maternal BMI & & & & $\begin{array}{c}1.08 \\
(1.01-1.14)\end{array}$ & 0.017 & $\begin{array}{c}1.17 \\
(1.13-1.22)\end{array}$ & $<0.001$ & $\begin{array}{c}1.27 \\
(1.20-1.34)\end{array}$ & $<0.001$ & $\begin{array}{c}1.17 \\
(1.11-1.23)\end{array}$ & $<0.001$ \\
\hline Paternal BMI & & & & $\begin{array}{c}1.03 \\
(0.97-1.11)\end{array}$ & 0.34 & $\begin{array}{c}1.15 \\
(1.10-1.20)\end{array}$ & $<0.001$ & $\begin{array}{c}1.21 \\
(1.13-1.31)\end{array}$ & $<0.001$ & $\begin{array}{c}1.11 \\
(1.05-1.18)\end{array}$ & $<0.001$ \\
\hline
\end{tabular}




\begin{tabular}{|c|c|c|c|c|c|c|c|c|c|}
\hline Gestational age & & $\begin{array}{c}0.93 \\
(0.84-1.03)\end{array}$ & 0.18 & $\begin{array}{c}0.99 \\
(0.92-1.07)\end{array}$ & 0.84 & $\begin{array}{c}0.92 \\
(0.80-1.06)\end{array}$ & 0.26 & $\begin{array}{c}0.96 \\
(0.87-1.06)\end{array}$ & 0.42 \\
\hline \multicolumn{10}{|l|}{ Birth weight } \\
\hline $\begin{array}{l}\text { Medium-low birth } \\
\text { weight }\end{array}$ & reference & - & & - & & - & & - & \\
\hline High birth weight & & $\begin{array}{c}1.62 \\
(0.83-3.16)\end{array}$ & 0.16 & $\begin{array}{c}0.98 \\
(0.57-1.67)\end{array}$ & 0.93 & $\begin{array}{c}1.66 \\
(0.73-3.77)\end{array}$ & 0.23 & $\begin{array}{c}0.86 \\
(0.42-1.76)\end{array}$ & 0.67 \\
\hline \multicolumn{10}{|l|}{ Breast feeding } \\
\hline$\geq 3$ months & reference & - & & - & & - & & - & \\
\hline$<3$ months & & $\begin{array}{c}1.02 \\
(0.61-1.71)\end{array}$ & 0.93 & $\begin{array}{c}1.18 \\
(0.81-1.72)\end{array}$ & 0.38 & $\begin{array}{c}1.33 \\
(0.64-2.75)\end{array}$ & 0.44 & $\begin{array}{c}0.99 \\
(0.62-1.57)\end{array}$ & 0.96 \\
\hline \multicolumn{10}{|l|}{$\begin{array}{l}\text { Evidence of puberty at } \\
10 \text { years }\end{array}$} \\
\hline No & reference & - & & - & & - & & - & \\
\hline Yes & & $\begin{array}{c}1.31 \\
(0.85-2.01)\end{array}$ & 0.23 & $\begin{array}{c}1.75 \\
(1.31-2.34)\end{array}$ & $<0.001$ & $\begin{array}{c}3.68 \\
(2.29-5.92)\end{array}$ & $<0.001$ & $\begin{array}{c}2.30 \\
(1.62-3.28)\end{array}$ & $<0.001$ \\
\hline \multicolumn{10}{|l|}{$\begin{array}{l}\text { Socioeconomic } \\
\text { position }\end{array}$} \\
\hline Medium-low & reference & - & & - & & - & & - & \\
\hline High & & $\begin{array}{c}0.85 \\
(0.57-1.24)\end{array}$ & 0.40 & $\begin{array}{c}0.75 \\
(0.57-0.98)\end{array}$ & 0.036 & $\begin{array}{c}0.50 \\
(0.28-0.88)\end{array}$ & 0.016 & $\begin{array}{c}0.90 \\
(0.64-1.28)\end{array}$ & 0.57 \\
\hline \multicolumn{10}{|l|}{ Maternal Education } \\
\hline Less than A-level & reference & - & & - & & - & & - & \\
\hline A-level or above & & $\begin{array}{c}0.94 \\
(0.59-1.53) \\
\end{array}$ & 0.85 & $\begin{array}{c}0.96 \\
(0.68-1.34) \\
\end{array}$ & 0.8 & $\begin{array}{c}0.82 \\
(0.40-1.67) \\
\end{array}$ & 0.59 & $\begin{array}{c}0.71 \\
(0.45-1.13) \\
\end{array}$ & 0.15 \\
\hline
\end{tabular}

Legend: $\mathrm{BMI}$ - Body mass index; $\mathrm{Cl}$ - Confidence interval; OW/OB - Overweight or obese; RRR - Relative risk ratio. 
In the fully adjusted model for males (table 4), both higher maternal and paternal BMI significantly increased the risk of childhood and early-adulthood onset of overweight/obesity compared to never being overweight/obese (RRR: 1.15-1.22; all $p<0.001)$. Early puberty also increased the risk of childhood (RRR: 9.14; 95\% Cl: 1.76-47.56) and earlyadulthood onset of overweight/obesity (RRR: 4.81; 95\% Cl: 1.14-20.32) compared with never being overweight/obese (both $p<0.04$ ). Additionally, having a high (versus normal-low) birth weight significantly increased the risk of early-adulthood onset of overweight/obesity (RRR: $2.67 ; 95 \% \mathrm{Cl}$ : 1.33-5.34), whereas having a higher gestational age significantly decreased the risk of early-adulthood onset of overweight/obesity (RRR: $0.88 ; 95 \% \mathrm{Cl}$ : 0.79-0.98) in relation to never being overweight/ obese (table 4). No association was found between trajectories of weight status and breastfeeding and maternal education in both sexes. 
Table 4. Associations between parental BMI and trajectories of weight status in males

\begin{tabular}{|c|c|c|c|c|c|c|c|c|c|c|c|}
\hline \multirow[b]{2}{*}{ Risk Factor } & \multirow[b]{2}{*}{$\begin{array}{c}\text { Included } \\
\mathrm{n}(\%)\end{array}$} & \multirow[b]{2}{*}{$\begin{array}{c}\text { Missing } \\
\text { n (\%) }\end{array}$} & \multirow{2}{*}{$\begin{array}{c}\text { Never } \\
\text { OW/OB } \\
\text { reference }\end{array}$} & \multicolumn{2}{|c|}{$\begin{array}{l}\text { Mid-adulthood onset } \\
\text { of OW/OB }\end{array}$} & \multicolumn{2}{|c|}{$\begin{array}{l}\text { Early-adulthood } \\
\text { onset of OW/OB }\end{array}$} & \multicolumn{2}{|c|}{$\begin{array}{c}\text { Childhood onset of } \\
\text { OW/OB }\end{array}$} & \multicolumn{2}{|c|}{ Other trajectories } \\
\hline & & & & $\begin{array}{c}\text { RRR } \\
(95 \% \mathrm{Cl})\end{array}$ & $p$ & $\begin{array}{c}\text { RRR } \\
(95 \% \mathrm{Cl})\end{array}$ & $p$ & $\begin{array}{c}\text { RRR } \\
(95 \% \mathrm{Cl})\end{array}$ & $p$ & $\begin{array}{c}\text { RRR } \\
(95 \% \mathrm{Cl})\end{array}$ & $p$ \\
\hline \multicolumn{12}{|l|}{$\begin{array}{l}\text { Unadjusted individual } \\
\text { models }\end{array}$} \\
\hline Maternal BMI & $\begin{array}{l}1,719 \\
(96.5)\end{array}$ & $\begin{array}{c}63 \\
(3.5)\end{array}$ & & $\begin{array}{c}1.05 \\
(0.97-1.13)\end{array}$ & 0.26 & $\begin{array}{c}1.16 \\
(1.10-1.22)\end{array}$ & $<0.001$ & $\begin{array}{c}1.23 \\
(1.16-1.31)\end{array}$ & $<0.001$ & $\begin{array}{c}1.09 \\
(1.03-1.16)\end{array}$ & $<0.001$ \\
\hline Paternal BMI & $\begin{array}{l}1,650 \\
(92.6)\end{array}$ & $\begin{array}{c}132 \\
(7.4)\end{array}$ & & $\begin{array}{c}1.05 \\
(0.96-1.15)\end{array}$ & 0.32 & $\begin{array}{c}1.17 \\
(1.10-1.23)\end{array}$ & $<0.001$ & $\begin{array}{c}1.27 \\
(1.17-1.37)\end{array}$ & $<0.001$ & $\begin{array}{c}1.15 \\
(0.99-1.15)\end{array}$ & $<0.001$ \\
\hline Mutually adjusted & $\begin{array}{l}1,628 \\
(91.4)\end{array}$ & $\begin{array}{l}154 \\
(8.6)\end{array}$ & & & & & & & & & \\
\hline Maternal BMI & & & & $\begin{array}{c}1.02 \\
(0.94-1.11)\end{array}$ & 0.56 & $\begin{array}{c}1.13 \\
(1.07-1.18)\end{array}$ & $<0.001$ & $\begin{array}{c}1.19 \\
(1.12-1.27)\end{array}$ & $<0.001$ & $\begin{array}{c}1.07 \\
(1.01-1.15)\end{array}$ & $<0.001$ \\
\hline Paternal BMI & & & & $\begin{array}{c}1.04 \\
(0.94-1.14)\end{array}$ & 0.44 & $\begin{array}{c}1.14 \\
(1.08-1.21)\end{array}$ & $<0.001$ & $\begin{array}{c}1.23 \\
(1.14-1.34)\end{array}$ & $<0.001$ & $\begin{array}{c}1.06 \\
(0.98-1.15)\end{array}$ & $<0.001$ \\
\hline $\begin{array}{l}\text { Adjusted for birth } \\
\text { factors, puberty and } \\
\text { socioeconomic position }\end{array}$ & $\begin{array}{l}1,140 \\
(64.0)\end{array}$ & $\begin{array}{c}642 \\
(36.0)\end{array}$ & & & & & & & & & \\
\hline Maternal BMI & & & & $\begin{array}{c}1.01 \\
(0.92-1.12)\end{array}$ & 0.8 & $\begin{array}{c}1.15 \\
(1.08-1.22)\end{array}$ & $<0.001$ & $\begin{array}{c}1.22 \\
(1.12-1.33)\end{array}$ & $<0.001$ & $\begin{array}{c}1.08 \\
(0.99-1.17)\end{array}$ & $<0.001$ \\
\hline Paternal BMI & & & & $\begin{array}{c}1.02 \\
(0.91-1.15)\end{array}$ & 0.71 & $\begin{array}{c}1.16 \\
(1.08-1.24)\end{array}$ & $<0.001$ & $\begin{array}{c}1.22 \\
(1.10-1.36)\end{array}$ & $<0.001$ & $\begin{array}{c}1.04 \\
(0.94-1.14)\end{array}$ & $<0.001$ \\
\hline
\end{tabular}




\begin{tabular}{|c|c|c|c|c|c|c|c|c|c|}
\hline Gestational age & & $\begin{array}{c}0.98 \\
(0.82-1.17)\end{array}$ & 0.84 & $\begin{array}{c}0.88 \\
(0.79-0.98)\end{array}$ & 0.016 & $\begin{array}{c}0.89 \\
(0.75-1.06)\end{array}$ & 0.20 & $\begin{array}{c}0.16 \\
(0.99-1.35)\end{array}$ & 0.06 \\
\hline \multicolumn{10}{|l|}{ Birth weight } \\
\hline $\begin{array}{l}\text { Medium-low birth } \\
\text { weight }\end{array}$ & reference & - & & - & & - & & - & \\
\hline High birth weight & & $\begin{array}{c}2.12 \\
(0.79-5.71)\end{array}$ & 0.14 & $\begin{array}{c}2.67 \\
(1.33-5.34)\end{array}$ & 0.006 & $\begin{array}{c}0.78 \\
(0.20-3.01)\end{array}$ & 0.72 & $\begin{array}{c}1.75 \\
(0.73-4.19)\end{array}$ & 0.21 \\
\hline \multicolumn{10}{|l|}{ Breast feeding } \\
\hline$\geq 3$ months & reference & - & & - & & - & & - & \\
\hline$<3$ months & & $\begin{array}{c}0.66 \\
(0.29-1.49)\end{array}$ & 0.32 & $\begin{array}{c}1.80 \\
(0.46-1.38)\end{array}$ & 0.42 & $\begin{array}{c}1.33 \\
(0.64-2.75)\end{array}$ & 0.44 & $\begin{array}{c}0.99 \\
(0.62-1.57)\end{array}$ & 0.24 \\
\hline \multicolumn{10}{|l|}{$\begin{array}{l}\text { Evidence of puberty at } \\
10 \text { years }\end{array}$} \\
\hline No & reference & - & & - & & - & & - & \\
\hline Yes & & $\begin{array}{c}2.56 \\
(0.35-18.64)\end{array}$ & 0.36 & $\begin{array}{c}4.71 \\
(1.11-19.95)\end{array}$ & 0.035 & $\begin{array}{c}9.21 \\
(1.77-48.02)\end{array}$ & 0.008 & $\begin{array}{c}2.92 \\
(0.52-16.36)\end{array}$ & 0.22 \\
\hline \multicolumn{10}{|l|}{$\begin{array}{l}\text { Socioeconomic } \\
\text { position }\end{array}$} \\
\hline Medium-low & reference & - & & - & & - & & - & \\
\hline High & & $\begin{array}{c}1.07 \\
(0.58-1.95)\end{array}$ & 0.83 & $\begin{array}{c}0.88 \\
(0.60-1.28)\end{array}$ & 0.51 & $\begin{array}{c}0.97 \\
(0.50-1.87)\end{array}$ & 0.93 & $\begin{array}{c}0.82 \\
(0.49-1.38)\end{array}$ & 0.46 \\
\hline \multicolumn{10}{|l|}{ Maternal Education } \\
\hline Less than A-level & reference & - & & - & & - & & - & \\
\hline A-level or above & & $\begin{array}{c}0.63 \\
(0.29-1.53)\end{array}$ & 0.23 & $\begin{array}{c}0.78 \\
(0.50-1.23)\end{array}$ & 0.29 & $\begin{array}{c}0.86 \\
(0.00-6.31)\end{array}$ & 0.72 & $\begin{array}{c}0.75 \\
(0.40-1.43)\end{array}$ & 0.39 \\
\hline
\end{tabular}

Legend: $\mathrm{BMI}$ - Body mass index; $\mathrm{Cl}$ - Confidence interval; OW/OB - Overweight or obese; RRR - Relative risk ratio. 
In post-hoc multivariable models using parental zBMI (instead of absolute BMI), maternal zBMI consistently showed stronger associations with childhood and early-adulthood onset of overweight/obesity than paternal zBMI in both males (RRR: 1.71 and 2.14 (respectively) for maternal, versus 1.55 and 1.85 for paternal zBMI; all $p<0.001$ ) and females (RRR: 1.85 and 2.50 for maternal, versus 1.53 and 1.80 for paternal zBMI; all $p<0.001)$

\section{Discussion}

This study sought to (i) describe the trajectories of weight status from childhood to mid-adulthood, and (ii) investigate the influence of parental BMI on weight status trajectories of their offspring. In both sexes, approximately $6 \%$ had childhood onset of overweight/obesity, and the vast majority of those overweight/obese in childhood remained overweight/obese in at least two of the three adulthood time-points considered in this study. Maternal and paternal BMI showed additive effects on offspring weight status trajectories across 32 years of the life course, independently of several confounding factors identified in the literature (Monasta et al., 2010; Parsons et al., 1999).

\section{Parental influence on weight status trajectories}

In both males and females, maternal and paternal BMI were positively associated with increased risk of childhood and early-adulthood onset of overweight/obesity (versus never being overweight/obese). In females, maternal and paternal BMI were also positively associated with increased risk of "other trajectories". This means that higher parental BMI also increased the risk of females being overweight/obese at $\geq 1$ time point between 10 and 42 years (apart from midadulthood onset of overweight/obesity). These results are consistent with the findings of the systematic review by Parsons et al., (1999), who reported that offspring of obese parents were consistently reported (in longitudinal studies) to be at risk of increased fatness from childhood into adulthood. In the fully adjusted models, these associations were slightly higher for maternal than paternal BMI in females (e.g. RRR for childhood onset of overweight/obesity: maternal $\mathrm{BMI}=1.27$, paternal $\mathrm{BMI}=1.21$ ), but similar for males (see tables 3 and 4). However, when repeating the analyses using parental zBMI, maternal zBMI consistently showed stronger associations than paternal zBMI with childhood and early adulthood onset of overweight/obesity than paternal $z B M I$ in both sexes. This suggests that the same shift (one zscore) in the distribution of parental BMI will have a higher influence in the offspring's weight status trajectories for maternal than paternal BMI.

The influence of parental BMI on offspring's overweight/obesity trajectories may operate through a variety of factors, including genetic predisposition, restricted/excessive intrauterine growth, shared environment and the inheritance of unhealthy behaviours (Monasta et al., 2010; Parsons et al., 1999). One hypothesis put forward for a higher risk of overweight/obesity in the offspring of overweight mothers is that such mothers are more likely not to breast-feed their infants and to be of lower SEP (Monasta et al., 2010), which may indirectly influence their child's BMI through factors like diet and physical activity. Given that maternal BMI remained significant after controlling for these two variables, our data does not support this hypothesis. Our results also do not support a possible mediating role of excessive intrauterine growth and infant feeding for the association of maternal BMI with offspring overweight/obesity (Monasta et al., 2010), as higher maternal BMI remained significantly associated with childhood and early-adulthood onset of overweight/obesity after adjusting for those variables. Although we have controlled for maternal education and childhood SEP (known confounders) (Monasta et al., 2010), the extent to which the observed influence of parental BMI on offspring's overweight/obesity trajectories is due to genetic, environmental or behavioural factors (or a combination of the three) cannot be assessed in the current study and requires investigation in future studies. Nevertheless, because (i) parental BMI was strongly associated with persistent overweight/ obesity, (ii) the population rates of overweight are high and (iii) it is potentially easier to intervene in parents than in children to reduce their overweight (Golan, 2006; Monasta et al., 2010), focusing on the family as a whole and targeting overweight parents in obesity interventions may be a successful strategy to reduce both adulthood overweight and 
offspring's risk of lifetime overweight/obesity at the population level. Additionally, as the influence of parental BMI may be largely biological (Monasta et al., 2010), it may be necessary to intervene in parents (particularly the mother) during or even before pregnancy.

\section{Other significant risk factors}

Early puberty was also a significant predictor of childhood and early adulthood onset of overweight/ obesity for both sexes, as well as belonging to "other trajectories" in females. These results are consistent with previous findings (Parsons et al., 1999; Prentice \& Viner, 2013), and improve upon existing literature which in general has lacked adjustment for SEP and parental fatness (Parsons et al., 1999). Because the available BCS70 data did not allow us to investigate whether obesity before 10 years may have led to early puberty and in turn to the subsequent onset of overweight/obesity, the possibility of reverse causality cannot be rejected and requires investigation in future studies. Nevertheless, the fact that early puberty was consistently associated with "early adulthood onset of overweight/obesity" (which by definition controls for overweight in childhood) indicates that this association between early puberty and subsequent adulthood overweight/obesity may be independent of preceding increased fatness, in line with the results of a recent systematic review by Prentice and Viner (2013).

Only three other factors emerged as significant predictors of weight status trajectories in the fully adjusted model, with mixed results between males and females. In males, higher gestational age significantly reduced the risk of having an early adulthood onset of overweight/obesity, in relation to never being overweight/obese. Although significant, the slight difference in the distribution of gestational age between sexes (39.6 weeks for males, 39.8 weeks for females; $p=0.02$ ) is unlikely to have influenced the lack of the same association for females. On the other hand, a birth-weight $\geq 4 \mathrm{~kg}$ significantly increased the risk of males having early adulthood onset of overweight/obesity (versus never being overweight/obese), an association previously reported in the literature (Monasta et al., 2010; Parsons et al., 1999). The higher prevalence of high birth-weight in males than in females $(11 \%$ versus $6 \% ; p<0.001)$, and the very large sample size of males in the early adulthood onset of overweight/obesity trajectory, may have influenced to some extent the ability to detect an association in males but not in females. Although the general consensus is that there is a consistent positive relationship between birth-weight and later obesity (Monasta et al., 2010; Parsons et al., 1999) and plausible mechanisms have been proposed (Parsons et al., 1999), the nature and strength of this association is less clear in studies that account for potential confounders, as in the present study. Both similar and reverse sex-differences in this association have been previously reported (Parsons et al., 1999), and more research is needed to clarify whether this association of birth-weight with later obesity is independent of known confounders (e.g. gestational diabetes, parental fatness and SEP).

The association of high childhood SEP with lower risk for childhood and early-adulthood onset of overweight/obesity only in females cannot be explained by differences in prevalence of high childhood SEP between sexes $(p=0.94)$. This sexinequality has been observed in previous studies using adulthood obesity as the outcome even after adjustment for potential adulthood cofounders (Heraclides et al., 2008; Khlat et al., 2009; Pudrovska et al., 2014). One potential explanation is that traditionally men tend to move upward on the social ladder, resulting in a closing of the social gap in health outcomes from childhood SEP; whereas women tend to remain in the social class of origin, magnifying the effect of parental social class on adulthood overweight (Heraclides et al., 2008). However, a considerable amount of literature has reported this effect of early life SEP in both sexes (Monasta et al., 2010; Parsons et al., 1999; Power et al., 2003). For example, Power et al. (2003) reported a significant association of early life social class with obesity at age 30 years for both males and females from the British 1958 birth cohort study. Among other factors, differences in sample sizes, timing and type of the childhood SEP variable, the number of potential confounders adjusted for, and using weight status at only one time-point (versus lifelong trajectories) as the outcome could explain the contradictory results. More studies adopting a life course approach, like the present study, and controlling for the main known confounders/mediators (e.g. childhood weight status, early puberty and parental BMI) are needed, 
to clarify whether a sex inequality exists for the SEP effect on later obesity or if it is a result of the selected data and methodology of different studies.

\section{Limitations and strengths}

This study had some limitations that deserve consideration. Only $47.4 \%$ of the eligible cohort members with valid $\mathrm{BMI}$ at 42 years were included for analysis and had weight status trajectories computed (due to participants missing BMI at $\geq 1$ time-points), which could have impacted on the results (e.g. reducing statistical power to detect some associations). However, there was no significant difference in parental BMI, gestational age and prevalence of high birth-weight between those included and excluded from analyses. All other variables were significantly different between those with and without complete data to compute trajectories of weight status, but the observed differences were very small (e.g. median $\mathrm{BMI}$ at 10 years: $16.7 \mathrm{~kg} / \mathrm{m}^{2}$ in included versus $16.3 \mathrm{~kg} / \mathrm{m}^{2}$ in excluded participants; high SEP: $34 \%$ in included versus $30 \%$ in excluded). Furthermore, differences between those included and excluded from the fully adjusted models were only significant in SEP (males and females), and breastfeeding in females; all other variables were similar between included and excluded participants ( $p>0.07$ for differences). There was a slightly higher proportion of participants with high SEP in those included versus excluded from the fully adjusted models (36\% versus $26 \%$ in males; $36 \%$ versus $29 \%$ in females). As sample sizes for each independent variable varied widely due to missing data and the observed differences were generally minor in size, it is unlikely that these differences would have had an impact on the results and their generalizability to the wider BCS70 cohort. As data were drawn from a national birth cohort, the findings are highly generalizable to the British population relatively contemporaneous in age to those in the BCS70 cohort. It is unclear how relevant the findings are to more recent age cohorts, as populations born in more recent years have been shown to have higher mean BMI and higher rates of overweight/obesity (Clarke, O'Malley, Johnston, \& Schulenberg, 2009; Stamatakis, Primatesta, Chinn, Rona, \& Falascheti, 2005). However, we would expect the effects of parental BMI on offspring overweight/obesity to be stronger in younger generations than that observed in the BCS70 cohort.

Weight and height at 10 years were measured but adulthood values were reported by participants, which may have led to biased BMI values and consequent classification of overweight/obesity. However, the recent review by Brisbois et al., (2012) reported overall good agreement between the results of studies using measured and reported adult BMI. Taking this into account and the strong significant associations found in the present study, it is unlikely that the possible misreporting of weight and height would significantly impact on the results seen for the association of parental BMI with persistent overweight/obesity and early adulthood onset of overweight/obesity.

The fact that trajectories of weight status were manually computed and not drawn from the data using a statistical procedure is both a strength and a limitation of the current study. Using the "manual process" for classification of trajectories of weight status allows us know exactly how each trajectory is characterised, and not rely on regression model specifications to derive weight status trajectories. However, there may have been other potential trajectories that were missed by, for example, joining the trajectories with $<5 \%$ prevalence in one only category (i.e. "other"). Using growth-mixture modelling (Muthén, 2001) with the five weight status trajectories identified in this study as the starting point, would (i) enable us to confirm whether the results from this study are confirmed when not using a manual procedure to derive the trajectories, (ii) include the 16-year sweep, as the model will be able to deal with the large amount of missing BMI data at this age, and (iii) address the issue of a reduced sample size in the fully adjusted regression models, by imputing the missing variables. We are currently undertaking this work. Other strengths of this study include investigating offspring weight status from childhood through to mid-adulthood in a large birth cohort sample, using a life course perspective (rather than only one timepoint in adulthood), which addresses two main limitations of many previous studies looking the association of parental BMI with offspring obesity (Parsons et al., 1999; Monasta et al., 2010). A final limitation was the fact that we did not include behavioural variables (such as diet and physical activity habits) which can influence participants' 
weight status at all ages, as well as their weight status trajectories.

\section{Conclusions}

In conclusion, higher paternal and maternal BMI showed additive influences on offspring weight status trajectories across 32 years of the life course after controlling for potential confounding factors identified in the literature (Parsons et al., 1999; Monasta et al., 2010). In particular, maternal and paternal BMI were significantly associated with higher risk of childhood and early-adulthood onset of overweight/obesity, and "other trajectories" (females only) versus never being overweight/ obese. Early puberty was also significantly associated with higher risk of childhood and early adulthood onset of overweight/obesity for both sexes. Other factors such as high SEP (females), gestational age and high birth-weight (males) also showed significant associations with childhood and early-adulthood onset of overweight/obesity, but these associations were weak and not consistent between sexes. The identified weight status trajectories highlight the importance of primary prevention, as most individuals remained overweight/obese after onset at any age and both childhood and adulthood obesity have been consistently associated with increased risks of type 2 diabetes, coronary heart disease and all-cause mortality later in life (Park et al., 2012; Park et al., 2013; Prospective Studies Collaboration, 2009). Early maturing overweight/obese children and those with overweight/obese parents are potential priority targets for interventions, due to their higher risk for persistent overweight/obesity through to mid-adulthood in both males and females. Prevention/intervention programmes should focus on the parents before pregnancy, or later on the family as a whole, since maternal and paternal BMI showed additive effects on offspring weight status trajectories from childhood to mid-adulthood.

\section{Acknowledgements}

The authors declare no competing interests. Permission for download and use of the BCS70 data was granted by the UK Data Service.

\section{References}

Brisbois, T. D., Farmer, A. P., \& McCargar, L. J. (2012). Early markers of adult obesity: A review. Obesity Reviews, 13(4), 347-367. http://dx.doi.org/10.1111/j.1467-789X.2011.00965.x

Centre for Longitudinal Studies. (2014). History of the 1970 British Cohort Study [Internet]. Retrieved 06/05/2014, from:

http://www.cls.ioe.ac.uk/page.aspx?\&sitesectionid=944\&sitesectiontitle=History+of+the+1970+Briti sh+Cohort+Study

Clarke, P., O'Malley, P. M., Johnston, L. D., \& Schulenberg, J. E. (2009). Social disparities in BMI trajectories across adulthood by gender, race/ethnicity and lifetime socio-economic position: 1986-2004. International Journal of Epidemiology, 38(2), 499-509. http://dx.doi.org/10.1093/ije/dyn214

Cole, T. J., Bellizzi, M. C., Flegal, K. M., \& Dietz, W. H. (2000). Establishing a standard definition for child overweight and obesity worldwide: International survey. British Medical Journal, 320(7244), 12401243. http://dx.doi.org/10.1136/bmj.320.7244.1240

Golan, M. (2006). Parents as agents of change in childhood obesity - from research to practice. International Journal of Pediatric Obesity, 1(2), 66-76. http://dx.doi.org/10.1080/17477160600644272

Health and Social Care Information Centre. (2014a). Statistics on obesity, physical activity and diet: England 2014 (pp. 1-101). London: Health and Social Care Information Centre.

Health and Social Care Information Centre. (2014b). National child measurement programme: England, 2013/14 school year (pp. 1-51). London: Health and Social Care Information Centre.

Heraclides, A., Witte, D., \& Brunner, E. J. (2008). The association between father's social class and adult obesity is not explained by educational attainment and an unhealthy lifestyle in adulthood. European Journal of Epidemiology, 23(8), 573-579. http://dx.doi.org/10.1007/s10654-008-9245-3

Khlat, M., Jusot, F., \& Ville, I. (2009). Social origins, early hardship and obesity: a strong association in women, but not in men? Social Science \& Medicine, 68(9), 1692-1699. http://dx.doi.org/10.1016/j.socscimed.2009.02.024 
Maes, H.H., Neale, M.C., Eaves, L.J. (1997). Genetic and environmental factors in relative body weight and human adiposity. Behavior Genetics, 27(4), 325-351. http://dx.doi.org/10.1023/A:1025635913927

Monasta, L., Batty, G. D., Cattaneo, A., Lutje, V., Ronfani, L., Van Lenthe, F. J., \& Brug, J. (2010). Early-life determinants of overweight and obesity: a review of systematic reviews. Obesity Reviews, 11(10), 695-708. http://dx.doi.org/10.1111/j.1467-789X.2010.00735.x

Muthén, B. (2001). Latent variable mixture modelling. In G.A. Marcoulides, \& R.E. Schumacker. (Eds.), New developments and techniques in structural equation modelling (pp. 1-33). Mahan, NJ: Lawrence Erlbaum Associates.

Office of Population Censuses and Surveys (1980). Classification of occupations 1980. London: Her Majesty's Stationery Office.

Park, M. H., Falconer, C., Viner, R. M., \& Kinra, S. (2012). The impact of childhood obesity on morbidity and mortality in adulthood: a systematic review. Obesity Reviews, 13(11), 985-1000. http://dx.doi.org/10.1111/j.1467-789X.2012.01015.x

Park, M. H., Sovio, U., Viner, R. M., Hardy, R. J., \& Kinra, S. (2013). Overweight in childhood, adolescence and adulthood and cardiovascular risk in later life: pooled analysis of three British birth cohorts. PLoS ONE, 8(7), e70684. http://dx.doi.org/10.1371/journal.pone.0070684

Parsons, T. J., Power, C., Logan, S., \& Summerbell, C. D. (1999). Childhood predictors of adult obesity: A systematic review. International Journal of Obesity and Related Metabolic Disorders, 23(Suppl 8), S1107.

Power, C., Manor, O., \& Matthews, S. (2003). Child to adult socioeconomic conditions and obesity in a national cohort. International Journal of Obesity, 27(9), 1081-1086. http://dx.doi.org/10.1038/sj.ijo.0802323

Prentice, P., \& Viner, R. M. (2013). Pubertal timing and adult obesity and cardiometabolic risk in women and men: a systematic review and meta-analysis. International Journal of Obesity, 37(8), 1036-1043. http://dx.doi.org/10.1038/ijo.2012.177

Prospective Studies Collaboration, Whitlock, G., Lewington, S., Sherliker, P., Clarke, R., Emberson, J., \& Peto, R. (2009). Body-mass index and cause-specific mortality in 900,000 adults: collaborative analyses of 57 prospective studies. Lancet, 373(9669), 1083-1096. http://dx.doi.org/10.1016/S01406736(09)60318-4

Pudrovska, T., Logan, E. S., \& Richman, A. (2014). Early-life social origins of later-life body weight: the role of socioeconomic status and health behaviors over the life course. Social Science Research, 46(2014), 59-71. http://dx.doi.org/10.1016/j.ssresearch.2014.02.007

Reilly, J. J., Methven, E., McDowell, Z. C., Hacking, B., Alexander, D., Stewart, L., \& Kelnar, C. J. (2003). Health consequences of obesity. Archives of Disease in Childhood, 88(9), 748-752. http://dx.doi.org/10.1136/adc.88.9.748

Stamatakis, E., Primatesta, P., Chinn, S., Rona, R., \& Falascheti, E. (2005). Overweight and obesity trends from 1974 to 2003 in English children: What is the role of socioeconomic factors? Archives of Disease in Childhood, 90(10), 999-1004. http://dx.doi.org/10.1136/adc.2004.068932

Venn, A. J., Thomson, R. J., Schmidt, M. D., Cleland, V. J., Curry, B. A., Gennat, H. C., \& Dwyer, T. (2007). Overweight and obesity from childhood to adulthood: A follow-up of participants in the 1985 Australian Schools Health and Fitness Survey. The Medical Journal of Australia, 186(9), 458-460.

World Health Organization (2011) Global status report on non-communicable diseases 2010. Geneva: World Health Organization. 
Appendix 1. Description and prevalence of possible overweight/obesity trajectories from childhood to mid-adulthood (overall and by sex)

\begin{tabular}{|c|c|c|c|c|c|c|c|}
\hline \multirow[b]{2}{*}{ Trajectory } & \multicolumn{4}{|c|}{ Age (years) } & \multirow{2}{*}{$\begin{array}{c}\text { Overall } \\
\mathrm{N}(\%)\end{array}$} & \multirow{2}{*}{$\begin{array}{c}\text { Females } \\
\mathbf{N}(\%)\end{array}$} & \multirow{2}{*}{$\begin{array}{l}\text { Males } \\
\mathbf{N}(\%) \\
\end{array}$} \\
\hline & 10 & $26-30$ & 34 & 42 & & & \\
\hline 1 & No & No & No & No & $1,230(29.5)$ & $962(40.2)$ & $268(15.0)$ \\
\hline 2 & No & No & No & $\mathrm{OW} / \mathrm{OB}$ & 334 (8.0) & 231 (9.7) & $103(5.8)$ \\
\hline 3 & No & No & OW/OB & $\mathrm{OW} / \mathrm{OB}$ & $233(5.6)$ & $145(6.1)$ & $88(4.9)$ \\
\hline 4 & No & OW/OB & OW/OB & $\mathrm{OW} / \mathrm{OB}$ & $1,617(38.7)$ & $588(24.6)$ & $1,029(57.7)$ \\
\hline 5 & $\mathrm{OW} / \mathrm{OB}$ & No & No & No & $27(0.7 \%)$ & $21(0.9)$ & $6(0.3)$ \\
\hline 6 & $\mathrm{OW} / \mathrm{OB}$ & OW/OB & No & No & $12(0.3)$ & $10(0.4)$ & $2(0.1)$ \\
\hline 7 & OW/OB & OW/OB & OW/OB & No & $14(0.3)$ & $11(0.5)$ & $3(0.2)$ \\
\hline 8 & $\mathrm{OW} / \mathrm{OB}$ & $\mathrm{OW} / \mathrm{OB}$ & OW/OB & $\mathrm{OW} / \mathrm{OB}$ & $258(6.2)$ & $148(6.2)$ & $110(6.2)$ \\
\hline 9 & No & $\mathrm{OW} / \mathrm{OB}$ & OW/OB & No & $55(1.3)$ & $32(1.0)$ & $23(1.3)$ \\
\hline 10 & $\mathrm{OW} / \mathrm{OB}$ & No & No & $\mathrm{OW} / \mathrm{OB}$ & $10(0.2)$ & $8(0.3)$ & $2(0.1)$ \\
\hline 11 & No & No & $\mathrm{OW} / \mathrm{OB}$ & No & $76(1.8)$ & $47(2.0)$ & $29(1.6)$ \\
\hline 12 & No & $\mathrm{OW} / \mathrm{OB}$ & No & No & $99(2.4)$ & $55(2.3)$ & $44(2.5)$ \\
\hline 13 & $\mathrm{OW} / \mathrm{OB}$ & OW/OB & No & OW/OB & $18(0.4)$ & $17(0.7)$ & $1(0.1)$ \\
\hline 14 & OW/OB & No & $\mathrm{OW} / \mathrm{OB}$ & OW/OB & $13(0.3)$ & $10(0.4)$ & $3(0.2)$ \\
\hline 15 & No & OW/OB & No & $\mathrm{OW} / \mathrm{OB}$ & $172(4.1)$ & $103(4.3)$ & 69 (3.9) \\
\hline 16 & OW/OB & No & OW/OB & No & $6(0.1)$ & $4(0.2)$ & $2(0.1)$ \\
\hline
\end{tabular}


Appendix 2. Results of unadjusted and adjusted (multivariable) multinomial logistic regression assessing the effect of maternal BMI on females' weight status trajectories.

\begin{tabular}{|c|c|c|}
\hline Risk Factor & $\begin{array}{l}\text { Included } \\
\text { n (\%) }\end{array}$ & $\begin{array}{l}\text { Missing } \\
\mathrm{n}(\%)\end{array}$ \\
\hline Unadjusted model & $\begin{array}{l}2,339 \\
(97.8)\end{array}$ & $53(2.2)$ \\
\hline \multicolumn{3}{|l|}{ Maternal BMI } \\
\hline $\begin{array}{l}\text { Adjusted for birth } \\
\text { factors }\end{array}$ & $\begin{array}{l}1,678 \\
(70.2)\end{array}$ & $\begin{array}{c}714 \\
(29.8)\end{array}$ \\
\hline \multicolumn{3}{|l|}{ Maternal BMI } \\
\hline \multicolumn{3}{|l|}{ Gestational age } \\
\hline \multicolumn{3}{|l|}{ Birth-weight } \\
\hline \multicolumn{3}{|l|}{ Birth-weight $\leq 4 \mathrm{~kg}$} \\
\hline \multicolumn{3}{|l|}{ Birth-weight >4 kg } \\
\hline \multicolumn{3}{|l|}{ Breast-feeding } \\
\hline \multicolumn{3}{|l|}{$\geq 3$ months } \\
\hline \multicolumn{3}{|l|}{$<3$ months } \\
\hline $\begin{array}{l}\text { Adjusted for birth } \\
\text { factors and puberty }\end{array}$ & $\begin{array}{l}1,672 \\
(69.9)\end{array}$ & $\begin{array}{c}720 \\
(30.1)\end{array}$ \\
\hline \multicolumn{3}{|l|}{ Maternal BMI } \\
\hline \multicolumn{3}{|l|}{ Gestational age } \\
\hline \multicolumn{3}{|l|}{ Birth-weight } \\
\hline \multicolumn{3}{|l|}{ Birth-weight $\leq 4 \mathrm{~kg}$} \\
\hline \multicolumn{3}{|l|}{ Birth-weight >4 kg } \\
\hline \multicolumn{3}{|l|}{ Breast-feeding } \\
\hline$\geq 3$ months & & \\
\hline$<3$ months & & \\
\hline
\end{tabular}

\begin{tabular}{c} 
Never \\
OW/OB \\
\hline reference
\end{tabular}

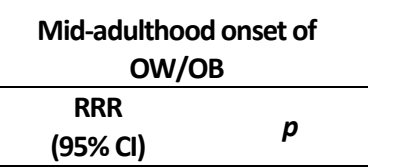

Early-adulthood onset of
OW/OB

\begin{tabular}{cc}
\multicolumn{2}{c}{ Childhood onset of OW/OB } \\
\hline $\begin{array}{c}\text { RRR } \\
(95 \% \mathrm{Cl})\end{array}$ \\
\hline
\end{tabular}

\begin{tabular}{cc}
\multicolumn{2}{c}{ Other trajectories } \\
\hline RRR \\
$(95 \% \mathrm{Cl})$ & $p$ \\
\hline
\end{tabular}

$1.10(1.05-1.15) \quad<0.001$

$1.21(1.17-1.25) \quad<0.001$

$1.31(1.25-1.37) \quad<0.001$

$1.17(1.13-1.22) \quad<0.001$

$1.08(1.02-1.15) \quad 0.007$

$1.20(1.15-1.24) \quad<0.001$

$1.31(1.24-1.38)$

$0.95(0.84-1.08)$

$<0.001$

$1.19(1.13-1.24)$

$<0.001$

$0.93(0.84-1.03) \quad 0.15$

$0.98(0.91-1.06) \quad 0.65$

$0.94(0.86-1.03)$

reference

$1.45(0.77-2.74) \quad 0.25$

$0.80(0.48-1.33)$

0.39

$1.13(0.52-2.47)$

0.75

$0.72(0.36-1.44)$

0.35

reference

$1.04(0.64-1.68)$

0.88

$1.28(0.91-1.80)$

0.16

$1.56(0.79-3.09)$

0.20

$1.02(0.67-1.57)$

0.92

reference

$1.08(1.02-1.14) \quad 0.01$

$1.19(1.14-1.23) \quad<0.001$

$1.30(1.24(1.37) \quad<0.001$

$1.18(1.12-1.23)<0.001$

reference

$0.93(0.84-1.03) \quad 0.15$

$0.98(0.91-1.05)$

0.57

$0.93(0.82-1.06)$

0.30

$0.93(0.85-1.02)$

0.14

reference

$1.45(0.77-2.75) \quad 0.25$

$0.81(0.49-1.34)$

0.41

$1.16(0.53-2.55)$

0.71

$0.73(0.36-1.47)$

0.37

reference

$1.04(0.64-1.69) \quad 0.86$

$1.32(0.93-1.87)$

0.12

$1.73(0.87-3.46)$

0.12

$1.09(0.71-1.70)$

0.69 
Evidence of puberty at

10 years

No

Yes

\section{Adjusted for birth}

\section{factors, puberty and}

socioeconomic

position

Maternal BMI

Gestational age

Birth-weight

Birth-weight $\leq 4 \mathrm{~kg}$

Birth-weight $>4 \mathrm{~kg}$

Breast-feeding

$\geq 3$ months

$<3$ months

Evidence of puberty at

10 years

No

Yes

Socio-economic

position

Medium-low

High

Maternal education

Less than A-level

A-level or above reference

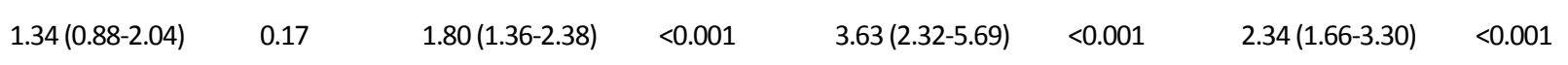

\section{$1,620 \quad 772$}

(67.7) (32.3)

\section{reference}

$1.07(1.01-1.14)$

$0.93(0.84-1.03)$

0.018

$1.18(1.13-1.22) \quad<0.001$

$0.98(0.91-1.06) \quad 0.60$

$1.29(1.22-1.36)$

0.92 (0.80-1.05)

$<0.001$

$1.17(1.12-1.23) \quad<0.001$

reference

reference

$1.54(0.79-2.98)$

0.20

$0.93(0.55-1.56)$

0.77

$1.53(0.69-3.43)$

0.30

$0.83(0.41-1.70)$

0.62

reference

$1.01(0.61-1.67)$

0.98

$1.20(0.83-1.72)$

0.33

$1.42(0.70-2.90)$

0.33

$0.98(0.62-1.54)$

0.92

reference

$1.35(0.88-2.06)$

0.17

$1.83(1.38-2.43) \quad<0.001$

$3.89(2.46-6.15)$

$<0.001$

$2.40(1.69-3.39)$

$<0.001$

reference

$0.86(0.59-1.26)$

0.44

$0.72(0.55-0.94)$

0.015

$0.48(0.28-0.82)$

0.008

$0.89(0.63-1.26)$

0.51

reference

Legend: $\mathrm{BMI}$ - Body mass index; $\mathrm{Cl}$ - Confidence interval; OW/OB - Overweight or obesity; RRR - Relative risk ratio. 
Appendix 3. Results of unadjusted and adjusted (multivariable) multinomial logistic regression assessing the effect of maternal BMI on males' weight status trajectories.

\begin{tabular}{|c|c|c|}
\hline Risk Factor & $\begin{array}{c}\text { Included } \\
\mathrm{n}(\%)\end{array}$ & $\begin{array}{c}\text { Missin } \\
\mathrm{n}(\%)\end{array}$ \\
\hline Unadjusted model & $\begin{array}{l}1,719 \\
(96.5)\end{array}$ & $63(3.5$ \\
\hline \multicolumn{3}{|l|}{ Maternal BMI } \\
\hline Adjusted for birth factors & $\begin{array}{l}1,231 \\
(69.1)\end{array}$ & $\begin{array}{c}551 \\
(30.9)\end{array}$ \\
\hline \multicolumn{3}{|l|}{ Maternal BMI } \\
\hline \multicolumn{3}{|l|}{ Gestational age } \\
\hline \multicolumn{3}{|l|}{ Birth-weight } \\
\hline \multicolumn{3}{|l|}{ Birth-weight $\leq 4 \mathrm{~kg}$} \\
\hline \multicolumn{3}{|l|}{ Birth-weight $>4 \mathrm{~kg}$} \\
\hline \multicolumn{3}{|l|}{ Breast-feeding } \\
\hline \multicolumn{3}{|l|}{$\geq 3$ months } \\
\hline \multicolumn{3}{|l|}{$<3$ months } \\
\hline $\begin{array}{l}\text { Adjusted for birth factors } \\
\text { and puberty }\end{array}$ & $\begin{array}{l}1,226 \\
(68.8)\end{array}$ & $\begin{array}{c}556 \\
(31.2)\end{array}$ \\
\hline \multicolumn{3}{|l|}{ Maternal BMI } \\
\hline \multicolumn{3}{|l|}{ Gestational age } \\
\hline \multicolumn{3}{|l|}{ Birth-weight } \\
\hline \multicolumn{3}{|l|}{ Birth-weight $\leq 4 \mathrm{~kg}$} \\
\hline \multicolumn{3}{|l|}{ Birth-weight $>4 \mathrm{~kg}$} \\
\hline \multicolumn{3}{|l|}{ Breast-feeding } \\
\hline \multicolumn{3}{|l|}{$\geq 3$ months } \\
\hline$<3$ months & & \\
\hline
\end{tabular}

\begin{tabular}{c} 
Never \\
OW/OB \\
\hline reference
\end{tabular}

\begin{tabular}{c}
$\begin{array}{c}\text { Mid-adulthood onset of } \\
\text { OW/OB }\end{array}$ \\
\hline RRR \\
$(95 \% \mathrm{Cl})$
\end{tabular}

\begin{tabular}{c}
$\begin{array}{c}\text { Early-adulthood onset of } \\
\text { OW/OB }\end{array}$ \\
\hline $\begin{array}{c}\text { RRR } \\
(95 \% \mathrm{Cl})\end{array} \quad p$ \\
\hline
\end{tabular}

Childhood onset of
OW/OB

\section{$1.05(0.97-1.13) \quad 0.26$}

$1.06(0.96-1.16)$

0.99 (0.84-1.17)

0.25

0.94

reference

$1.96(0.73-5.22)$

0.18

reference

0.65 (0.31-1.34)

0.25

$1.06(0.96-1.16)$

$0.99(0.84-1.17)$

0.23

0.93

reference

$1.95(0.73-5.20)$

0.18

reference

0.83

$0.64(0.31-1.34)$
0.24

\section{$1.16(1.10-1.22) \quad<0.001$}

$1.19(1.12-1.26)$

$0.87(0.79-0.96)$

$<0.001$

0.007

$1.26(1.16-1.36) \quad<0.001$

$0.89(0.75-1.05) \quad 0.15$

$1.12(1.04-1.21) \quad 0.004$

$1.13(0.98-1.30)$

11

0.002

$0.84(0.22-3.23) \quad 0.81$

$1.81(0.77-4.26)$

0.17

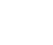

-

$1.10(0.44-2.72) \quad 0.84$

$0.73(0.38-1.39)$

0.34

$1.19(1.12-1.27) \quad<0.001$

$1.26(1.16-1.37) \quad<0.001$

$0.89(0.75-1.05) \quad 0.16$

$1.11(1.03-1.20) \quad 0.008$

$0.87(0.79-0.97) \quad 0.009$

(2)

$2.92(1.47-5.79) \quad 0.002$

$0.81(0.21-3.12)$

0.77

$1.79(0.76-4.22)$

0.18

$0.94(0.57-1.55)$

0.80 
Evidence of puberty at 10

years

No

Yes

\section{Adjusted for birth}

factors, puberty and $\quad 1,181 \quad 601$

socioeconomic position (66.3) (33.7)

Maternal BMI

Gestational age

Birth weight

Birth-weight $\leq 4 \mathrm{~kg}$

Birth-weight $>4 \mathrm{~kg}$

Breast-feeding

$\geq 3$ months

$<3$ months

Evidence of puberty at 10

years

Yes

No

Socio-economic position

Medium-low

High

reference

$2.34(0.32-$

16.99)

0.40

4.80 (1.14-

20.19)

0.032

8.86 (1.72-

45.66)

0.009

$2.80(0.50$

15.62)

0.24

reference

$1.04(0.95-1.15) \quad 0.40$

$1.18(1.11-1.25)<0.001$

$1.26(1.16-1.37)<0.001$

$1.09(1.01-1.18) \quad 0.03$

$1.00(0.84-1.18) \quad 0.96$

$0.88(0.79-0.98) \quad 0.016$

$0.90(0.76-1.07) \quad 0.22$

$1.16(1.0-1.35) \quad 0.05$

reference

$2.01(0.75-5.37)$

0.17

$2.86(1.44-5.68) \quad 0.003$

$0.80(0.21-3.07) \quad 0.74$

$1.83(0.77-4.32)$

0.17

reference

$0.66(0.30-1.46)$

0.31

$0.85(0.50-1.43) \quad 0.53$

$0.93(0.36-2.37) \quad 0.87$

$0.64(0.32-1.26)$

0.20

reference

-

$0.37 \quad 4.70(1.11$

$19.85)$

0.035

$9.12(1.76-$

47.22)

0.008

2.89 (0.52-

18.22)

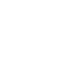

reference

$1.05(0.58-1.89)$

0.88

$0.82(0.67-1.18)$

0.28

$0.86(0.45-1.65) \quad 0.66$

$0.75(0.45-1.25)$

0.27

Maternal education

Less than A-level

reference

A-level or above

$\begin{array}{llll}0.69(0.33-1.45) & 0.33 & 0.81(0.52-1.26) & 0.34\end{array}$

$0.87(0.39-1.94)$

0.73

$0.74(0.39-1.41)$

0.36 
Appendix 4. Results of unadjusted and adjusted (multivariable) multinomial logistic regression assessing the effect of paternal BMI on females' weight status trajectories.

\begin{tabular}{|c|c|c|}
\hline Risk Factor & $\begin{array}{c}\text { Included } \\
\mathrm{n}(\%)\end{array}$ & $\begin{array}{c}\text { Missing } \\
\mathrm{n}(\%)\end{array}$ \\
\hline Unadjusted model & $\begin{array}{l}2,250 \\
(94.1)\end{array}$ & $\begin{array}{l}142 \\
(5.9)\end{array}$ \\
\hline \multicolumn{3}{|l|}{ Paternal BMI } \\
\hline Birth factors & $\begin{array}{l}1,629 \\
(68.1)\end{array}$ & $\begin{array}{c}763 \\
(31.9)\end{array}$ \\
\hline \multicolumn{3}{|l|}{ Paternal BMI } \\
\hline \multicolumn{3}{|l|}{ Gestational age } \\
\hline \multicolumn{3}{|l|}{ Birth-weight } \\
\hline \multicolumn{3}{|l|}{ Birth-weight $\leq 4 \mathrm{~kg}$} \\
\hline \multicolumn{3}{|l|}{ Birth-weight $>4 \mathrm{~kg}$} \\
\hline \multicolumn{3}{|l|}{ Breast- feeding } \\
\hline \multicolumn{3}{|l|}{$\geq 3$ months } \\
\hline \multicolumn{3}{|l|}{$<3$ months } \\
\hline $\begin{array}{l}\text { Adjusted for birth } \\
\text { factors and puberty }\end{array}$ & $\begin{array}{l}1,625 \\
(67.9)\end{array}$ & $\begin{array}{c}767 \\
(32.1)\end{array}$ \\
\hline \multicolumn{3}{|l|}{ Paternal BMI } \\
\hline \multicolumn{3}{|l|}{ Gestational age } \\
\hline \multicolumn{3}{|l|}{ Birth-weight } \\
\hline \multicolumn{3}{|l|}{ Birth-weight $\leq 4 \mathrm{~kg}$} \\
\hline \multicolumn{3}{|l|}{ Birth-weight $>4 \mathrm{~kg}$} \\
\hline \multicolumn{3}{|l|}{ Breast-feeding } \\
\hline$\geq 3$ months & & \\
\hline$<3$ months & & \\
\hline
\end{tabular}

\begin{tabular}{c} 
Never \\
OW/OB \\
\hline reference
\end{tabular}

\begin{tabular}{c} 
Mid-adulthood onset of \\
OW/OB \\
\hline RRR \\
$(95 \% \mathrm{Cl})$
\end{tabular}

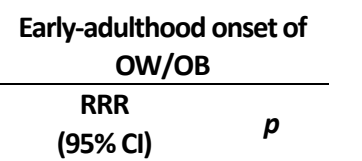

\begin{tabular}{c} 
Childhood onset of \\
OW/OB \\
\hline RRR $\quad p$ \\
$(95 \% \mathrm{Cl}) \quad p$ \\
\hline
\end{tabular}

\begin{tabular}{c}
\multicolumn{2}{c}{ Other trajectories } \\
\hline $\begin{array}{c}\text { RRR } \\
(95 \% \mathrm{Cl})\end{array} \quad p$ \\
\hline
\end{tabular}

$1.09(1.03-1.15) \quad 0.002$

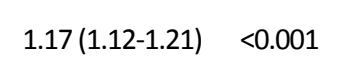

$1.05(0.98-1.12) \quad 0.14$

$1.18(1.13-1.23)<0.001$

$1.27(1.19-1.37)<0.001$

$1.15(1.09-1.21)<0.001$

$0.93(0.83-1.03) \quad 0.14$

$1.00(0.93-1.07) \quad 0.92$

$0.94(0.83-1.07) \quad 0.38$

$0.96(0.87-1.05) \quad 0.36$

reference

$1.56(0.83-2.95) \quad 0.17$

$0.97(0.59-1.60) \quad 0.91$

$1.69(0.80-3.57) \quad 0.17$

$0.82(0.41-1.64) \quad 0.58$

reference

$1.09(0.67-1.78) \quad 0.73$

$1.24(0.88-1.74) \quad 0.23$

$1.25(0.65-2.38) \quad 0.50$

$1.04(0.68-1.60)$

0.86

$1.05(0.98-1.12) \quad 0.17$

$1.17(1.12-1.22) \quad<0.001$

$1.25(1.17-1.34)<0.001$

$1.13(1.07-1.20) \quad<0.001$

$0.92(0.83-1.24) \quad 0.13$

$0.99(0.92-1.07) \quad 0.84$

$0.93(0.81-1.06) \quad 0.27$

$0.95(0.86-1.04) \quad 0.28$

reference

$1.57(0.83-2.97) \quad 0.17$

$0.98(0.59-1.62) \quad 0.93$

$1.75(0.82-3.72) \quad 0.15$

$0.84(0.42-1.68)$

0.63

reference

$1.10(0.67-1.80) \quad 0.70$

$1.30(0.92-1.84) \quad 0.14$

$1.37(0.71-2.65) \quad 0.34$

$1.13(0.73-1.75)$

0.59 
Evidence of puberty at

10 years

Yes

\section{Adjusted for birth}

factors, puberty and $\quad 1,578 \quad 814$

socioeconomic position (66.0) (34.0)

Paternal BMI

Gestational age

Birth-weight

Birth-weight $\leq 4 \mathrm{~kg}$

Birth-weight $>4 \mathrm{~kg}$

Breast-feeding

$\geq 3$ months

$<3$ months

Evidence of puberty at

10 years

No

Yes

Socio-economic position

Medium-low

High

Maternal education

Less than A-level

A-level or above reference

$$
1.32(0.87-2.02) \quad 0.19
$$

$1.81(1.37-2.40) \quad<0.001$

$3.69(2.35-5.78) \quad<0.001$

$2.36(1.67-3.33) \quad<0.001$

$1.04(0.97-1.11) \quad 0.28$

$0.93(0.83-1.03) \quad 0.16$

$1.16(1.11-1.22)<0.001$

$1.25(1.16-1.34)<0.001$

$0.92(0.80-1.05) \quad 0.19$

$1.13(1.06-1.20)<0.001$

$0.99(0.92-1.07) \quad 0.78$

$0.96(0.87-1.06)$

0.45

reference

$1.68(0.86-3.26) \quad 0.13$

$1.13(0.67-1.90) \quad 0.65$

$2.30(1.05-5.00) \quad 0.036$

$0.98(0.48-1.98)$

0.95

reference

$1.04(0.62-1.75) \quad 0.88$

$1.18(0.82-1.70)$

0.37

$1.14(2.41-6.02) \quad<0.001$

$1.00(0.63-1.58)$

1

reference

$1.32(0.86-2.03)$

0.2

$1.83(1.37-2.43) \quad<0.001$

$3.81(2.41-6.02) \quad<0.001$

$2.41(1.70-3.41) \quad<0.001$

reference

$0.80(0.54-1.17) \quad 0.24$

$0.66(0.51-0.87) \quad 0.003$

$0.42(0.25-0.73) \quad 0.002$

$0.81(0.57-1.14) \quad 0.22$

reference

Legend: $\mathrm{BMI}$ - Body mass index; $\mathrm{Cl}$ - Confidence interval; OW/OB - Overweight or obesity; RRR - Relative risk ratio. 
Appendix 5. Results of unadjusted and adjusted (multivariable) multinomial logistic regression assessing the effect of paternal BMI on males' weight status trajectories

\begin{tabular}{|c|c|c|}
\hline Risk Factor & $\begin{array}{l}\text { Included } \\
\mathrm{n}(\%)\end{array}$ & $\begin{array}{l}\text { Missing } \\
\mathrm{n}(\%)\end{array}$ \\
\hline Unadjusted model & $\begin{array}{l}1,650 \\
(92.6)\end{array}$ & $\begin{array}{l}132 \\
(7.4)\end{array}$ \\
\hline \multicolumn{3}{|l|}{ Paternal BMI } \\
\hline Adjusted for birth factors & $\begin{array}{l}1,190 \\
(66.8)\end{array}$ & $\begin{array}{c}592 \\
(33.2)\end{array}$ \\
\hline \multicolumn{3}{|l|}{ Paternal BMI } \\
\hline \multicolumn{3}{|l|}{ Gestational age } \\
\hline \multicolumn{3}{|l|}{ Birth-weight } \\
\hline \multicolumn{3}{|l|}{ Birth-weight $\leq 4 \mathrm{~kg}$} \\
\hline \multicolumn{3}{|l|}{ Birth-weight >4 kg } \\
\hline \multicolumn{3}{|l|}{ Breast-feeding } \\
\hline \multicolumn{3}{|l|}{$\geq 3$ months } \\
\hline \multicolumn{3}{|l|}{$<3$ months } \\
\hline $\begin{array}{l}\text { Adjusted for birth factors } \\
\text { and puberty }\end{array}$ & $\begin{array}{l}1,185 \\
(66.5)\end{array}$ & $\begin{array}{c}597 \\
(33.5)\end{array}$ \\
\hline \multicolumn{3}{|l|}{ Paternal BMI } \\
\hline \multicolumn{3}{|l|}{ Gestational age } \\
\hline \multicolumn{3}{|l|}{ Birth-weight } \\
\hline \multicolumn{3}{|l|}{ Birth-weight $\leq 4 \mathrm{~kg}$} \\
\hline \multicolumn{3}{|l|}{ Birth-weight $>4$ kg } \\
\hline \multicolumn{3}{|l|}{ Breast-feeding } \\
\hline$\geq 3$ months & & \\
\hline$<3$ months & & \\
\hline
\end{tabular}

\begin{tabular}{c} 
Never \\
OW/OB \\
\hline reference
\end{tabular}

\begin{tabular}{c}
$\begin{array}{c}\text { Mid-adulthood onset of } \\
\text { OW/OB }\end{array}$ \\
\hline RRR \\
$(95 \% \mathrm{Cl})$
\end{tabular}

Early-adulthood onset of

\begin{tabular}{cc}
\multicolumn{2}{c}{ OW/OB } \\
\hline RRR \\
$(95 \% \mathrm{Cl})$ & $p$
\end{tabular}

$1.05(0.96-1.15) \quad 0.32$

$1.17(1.10-1.23) \quad<0.001$

$1.03(0.93-1.15) \quad 0.59$

$0.98(0.83-1.16) \quad 0.82$

$1.19(1.11-1.27) \quad<0.001$

$0.87(0.79-0.97) \quad 0.01$

$1.28(1.16-1.42)$

$0.89(0.76-1.06)$

$<0.001$

0.19

$1.07(0.98-1.17)$

$1.11(0.96-1.29)$

0.16

reference

$2.08(0.78-5.56) \quad 0.15$

$2.97(1.50-5.89) \quad 0.002$

$0.83(0.22-3.18)$

0.79

$1.79(0.75-4.27)$

0.19

reference

$$
0.68(0.32-1.44) \quad 0.32
$$

0.97 (0.59-1.59)

0.89

$0.95(0.40-2.28)$

0.92

$1.80(0.41-1.55)$

0.50

$1.03(0.92-1.14) \quad 0.64$

$1.18(1.11-1.27)<0.001$

$1.28(1.16-1.41)$

$0.90(0.76-1.06)$

$<0.001$

$1.06(0.97-1.16)$

$1.12(0.97-1.30)$

0.19

$0.98(0.83-1.16) \quad 0.81$

$0.87(0.79-0.97) \quad 0.01$

reference

$2.07(0.77-5.54) \quad 0.15$

$2.87(1.45-5.70) \quad 0.003$

$0.81(0.21-3.09)$

0.75

$1.76(0.74-4.20)$

0.20

reference

$0.68(0.32-1.43) \quad 0.31$

$0.97(0.58-1.59)$

0.89

$0.93(0.39-2.22)$

0.87

$0.84(0.43-1.64)$

0.61 
Evidence of puberty at 10

years

No

Yes

Adjusted for birth factors,

puberty and socioeconomic $1,150 \quad 632$

$\begin{array}{lll}\text { puberty and socioeconomic } & (64.5) \quad(35.5) \\ \text { position }\end{array}$

Gestational age

Birth-weight

Birth-weight $\leq 4 \mathrm{~kg}$

Birth-weight $>4 \mathrm{~kg}$

Breast-feeding

$\geq 3$ months

$<3$ months

Evidence of puberty at 10

years

No

Yes

Socio-economic position

Medium-low

High

Maternal education

Less than A-level reference

$\begin{array}{llllllll}2.39(0.33-17.37) & 0.39 & 4.77(1.13-20.09) & 0.033 & 8.83(1.71(45.58) & 0.009 & 3.65(0.69-19.18) & 0.13\end{array}$

$1.02(0.91-1.14) \quad 0.69 \quad 1.18(1.10-1.26) \quad<0.001$

$1.25(1.13-1.39) \quad<0.001 \quad 1.05(0.95-1.15) \quad 0.32$

$0.97(0.82-1.16) \quad 0.77$

$0.87(0.79-0.97) \quad 0.012$

$0.90(0.76-1.07) \quad 0.25$

$1.15(0.98-1.34) \quad 0.08$

reference

$2.19(0.81-5.88) \quad 0.12$

$2.88(1.45-5.74) \quad 0.003$

$0.83(0.22-3.19) \quad 0.79$

$1.80(0.75-4.31) \quad 0.19$

reference

$0.70(0.31-1.57) \quad 0.38$

$0.89(0.52-1.50) \quad 0.66$

$0.82(0.33-2.01) \quad 0.66$

$0.72(0.36-1.45)$

0.37

reference

$2.60(0.36-18.96) \quad 0.35$

$4.74(1.12-20.02) \quad 0.034$

$9.07(1.75-46.91) \quad 0.009$

$3.72(0.71-19.62)$

0.12

reference

$1.10(0.60-2.01) \quad 0.75$

$0.85(0.59-1.23) \quad 0.38$

$0.93(0.49-1.77)$

0.82

$0.83(0.50-1.40)$

0.49

reference 